EPJ Web of Conferences 77, 00008 (2014)

DOI: $10.1051 /$ epjconf / 20147700008

(c) Owned by the authors, published by EDP Sciences, 2014

\title{
Dynamic force calibration by impact excitation
}

\author{
Meng Feng ${ }^{1}$, Zhang ZhiMin ${ }^{1}$ Zhang Yue ${ }^{1}$ and Zhang $\mathrm{Wei}^{1}$ \\ ${ }^{1}$ National institute of metrology, Mechanics and Acoustics Division, China
}

\begin{abstract}
Introduce a method of dynamic force calibration by impact excitation. An analysis of the $200 \mathrm{kN}$ standard dynamic force calibration equipment at NIM is presented. The heavy hammer and the force transducer under test are brought to collision, and the impact force is measured by laser-Doppler interferometer. Traceability of force is realized by the determination of mass and acceleration. Two experiments were introduced and a typical example of impact process was performed.
\end{abstract}

\section{Introduction}

In recent years, the demand of dynamic force calibration has increased due to the rising number of dynamic applications and due to improved safety standards, e.g. in the automotive industry. More and more dynamic force transducers are used. The measurement of dynamic force develops rapidly and has made great progress. More and more people have convinced that the force transducer which was used in dynamic force measurement should be carried out not only the static calibration but also the dynamic calibration. By the dynamic calibration we can get the dynamic characteristics of transducer or the measuring system. Dynamic force calibrating procedures have been developed many years before at PTB and NIM force $1 a b^{[1][2][3]}$.

\section{Realisation of equipment}

National Institute of Metrology (NIM) has developed two sets of dynamic force standard equipment. The old equipment (Figue1) is fall hammer type dynamic force calibration equipment, which can generate the bell shape force pulse (Gauss pulse). The specifications of the equipment are: force measuring range: $500 \mathrm{~N}-1 \mathrm{MN}$, force rising time: $\geqslant 0.3 \mathrm{~ms}$, expanded uncertainty: $3 \%(\mathrm{k}=3)$. The dynamic force acting on the force transducer is traceable to mass and acceleration by $F=m a$, where $m$ is the mass of hammer and $a$ is the impact acceleration of hammer. Original we measure the impact acceleration of drop hammer by a standard accelerometer. The new equipment was shown in the figure 2 . We measure the acceleration by a laser Interferometer. Traceability for force is realized via

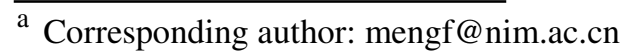

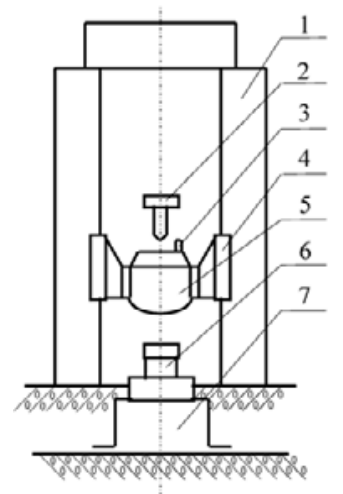

1. Tracks 2.releasing mechanism 3. Standard accelerometer 4. Guiders 5.hammer 6.dynamic force transducer 7.anvil

Fig.1. Standard dynamic force equipment

the measurement of acceleration with laser-Dopplerinterferometers and the determination of the dropping masses. The specifications of new equipment are: force measuring range: $200 \mathrm{~N}-200 \mathrm{kN}$, force rising time: $\geqslant$ $0.3 \mathrm{~ms}$, expanded uncertainty: $2 \%(\mathrm{k}=3)^{[2]}$. The $200 \mathrm{KN}$ dynamic force equipment use drop hammer structure. We measure the acceleration of drop hammer by laser interferometer. And then we get the impact force generated by drop hammer. In this paper we focus on the new equipment.

\subsection{Main structure and working principle}

The new 200kN standard equipment consists of anvil, frame, track, hammer, guiders, lifting and releasing mechanism, references dynamic force transducer and the laser-Doppler-interferometer. An LDI head (Polytec OFV550) mounted on top of the equipment, which can be adjusted easily. As shown in Fig.2.The new equipment 
has two hammers to adapt to different range. One is two kilogram, the other is twenty kilogram. The hammer can be upgraded by electromagnet automatic. In order to ensure the whereabouts of the hammer is ideal freefalling. We adjust the level of drop hammer and base level strictly. The gap of hammer and tracks is designed suitable. The hammer is made by steel forgings. Has uniform quality. The surface of hammer is made a large radius of curvature of the arc shape, can make the sensing on the impact force has a preferably neutral, thus ensuring smooth, impact force waveform and a higher frequency response. In order to avoiding the effect of structure vibration, the laser interferometer is installed on the top of the equipment, and independent of frame structure. Hammer fall freely acting on the transducer under tested. The laser interferometer measurement system and the transducer system to be calibrated simultaneously responding, and record the impact force value. The bottom of the equipment is the massive rigid anvil, which is fixed on the ground separate from the frame structure, to avoid the laser measure system affected by hammer impact.

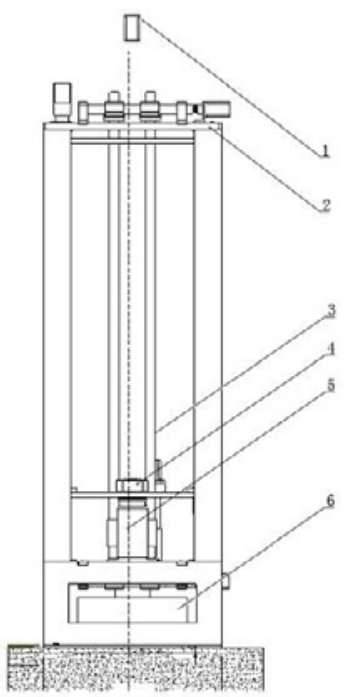

1. Laser-Doppler-interferometer

2. Servo Motor 3. Tracks 4. Electromagnet 5. Hammer 6. Anvil

Fig.2. Standard dynamic force equipment

The impact force calculated by formula (1).

$$
F=m a
$$

\subsection{Control and signal process system}

Control and signal process system is consisting of PXI1042 host, PXI-5122 digitizing oscilloscope instrument and PXI-6225 high-speed data acquisition card. The PXI5122 is a high-performance digital oscilloscope instrument performance for maximum 100M sampling rate, 14bit data acquisition function. By PXI-5122 we can record and analysis the signal of laser interferometer decoder. The displacement and limit sensor in system are processed by PXI-6225. The four servo motors are controlled by the host computer trough the serial port.

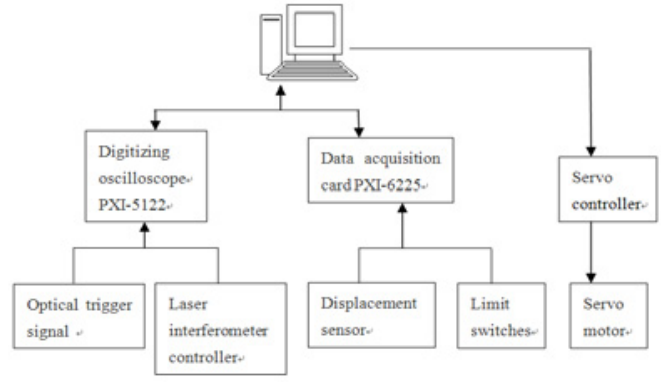

Fig .3. Control and data analysis system

\subsection{Laser interferometer system}

Laser interferometer system is consisting of OFV-505 laser head and OFV-5000 controller, the VD-09 velocity decoder inside, which decoding the original laser Doppler signal to velocity signal. Laser head is OFV-505 laser head, using an improved Mach-Zehnderinterferometer (Mach-ZehnderInterferometer).

\section{Principle of dynamic force measurement by laser}

The laser interferometer OFV-505 and OFV-5000 use the principle of the heterodyne interferometer to acquire the characteristics of mechanical vibrations or transient motion processes. As fig 4 shows.

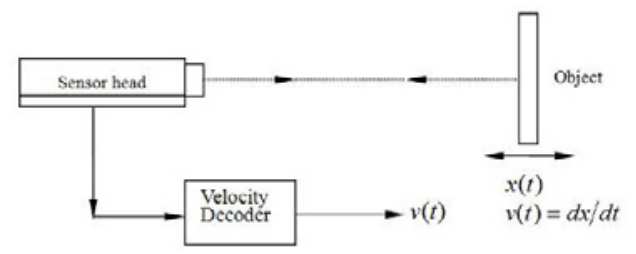

Fig .4.Signal in the interferometer

With this type of interferometer, a high-frequency carrier signal is generated on the photo detector with the aid of a Bragg cell. With signal processing system, the phase sequence can be got by demodulating the discrete-time sequence of interferometer generated orthogonal signals. And then calculate the displacement sequence, then through a series of processing can eventually get the time series of acceleration. Fig 5 shows.

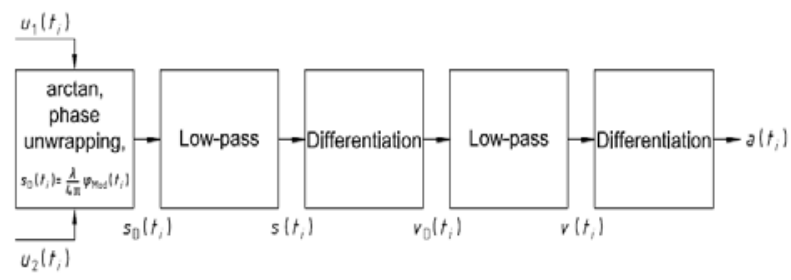

Fig .5. Schematic of laser acceleration process Where:

$$
\varphi_{\text {mod }}\left(t_{i}\right)=\arctan \frac{u_{2}\left(t_{i}\right)}{u_{1}\left(t_{i}\right)}+n \pi
$$


The velocity and acceleration can be caculated by formuler (3) and (4).

$$
\begin{aligned}
& v_{D}\left(t_{i}\right)=\frac{1}{\Delta t}\left(s\left(t_{i}\right)-s\left(t_{i-1}\right)\right) \\
& a\left(t_{i}\right)=\frac{1}{2 \Delta t}\left(v\left(t_{i}\right)-v\left(t_{i-1}\right)\right)
\end{aligned}
$$

After we get the time series of acceleration,we can get the time series of force $F\left(t_{i}\right)$ by formuler (1). So $F_{\text {peak }}$ is the maximum of force time series. as the fig 6 shown. The pulse curve is acceleration change process curve. The step curve is velocity change process curve.

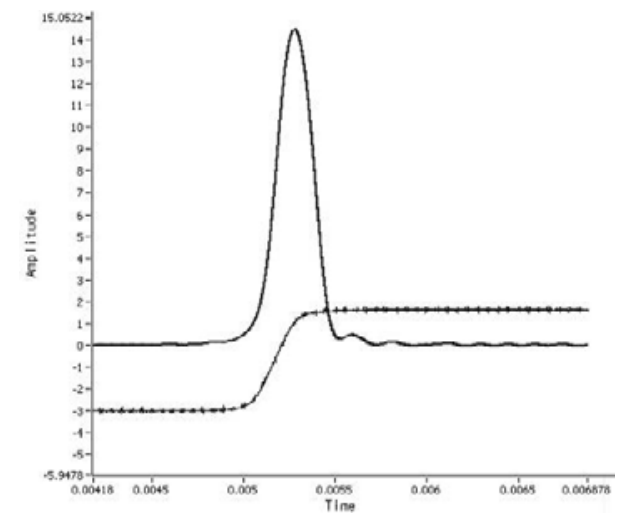

Fig.6. Impact velocity and acceleration during the change process

\section{Experiments}

We have done two main experiments using impact excite force equipment.

\subsection{Comparison of accelerator measurement and laser measurement}

We mount the standard accelerometer on the top of the standard hammer as fig 6 shows. It measures the same impact process with laser-interferometer. While the data acquisition system record the signal of impact process from laser interferometer accelerometer simultaneously. The results are shown in table (1).

Table 1

\begin{tabular}{|c|l|c|c|c|c|}
\hline $\begin{array}{c}\text { Test } \\
\text { No }\end{array}$ & \multicolumn{2}{|c|}{$\begin{array}{c}\text { Force measured by } \\
\text { laser-Doppler- } \\
\text { interferometer }\end{array}$} & \multicolumn{2}{|c|}{$\begin{array}{c}\text { Force measured } \\
\text { by accelerometer }\end{array}$} & $\begin{array}{l}\text { Relative } \\
\text { Error } \\
(\%)\end{array}$ \\
\cline { 2 - 5 } & $\begin{array}{l}\text { Force } \\
(\mathrm{kN})\end{array}$ & $\begin{array}{l}\text { Rising } \\
\text { time(ms) }\end{array}$ & $\begin{array}{l}\text { Force } \\
(\mathrm{kN})\end{array}$ & $\begin{array}{l}\text { Rising } \\
\text { time(m) }\end{array}$ & \\
\hline 1 & 2.6911 & 0.897 & 2.708 & 0.820 & -0.6 \\
2 & 2.4558 & 0.898 & 2.466 & 0.860 & -0.4 \\
3 & 2.7621 & 0.846 & 2.774 & 0.840 & -0.4 \\
4 & 3.2598 & 0.743 & 3.282 & 0.754 & -0.7 \\
5 & 3.0051 & 0.821 & 3.004 & 0.794 & 0.1 \\
6 & 3.5928 & 0.692 & 3.596 & 0.704 & -0.1 \\
7 & 4.9813 & 0.564 & 4.996 & 0.570 & -0.3 \\
8 & 4.8712 & 0.589 & 4.898 & 0.578 & -0.6 \\
9 & 4.9812 & 0.590 & 5.000 & 0.564 & -0.4 \\
10 & 5.3384 & 0.501 & 5.364 & 0.540 & -0.5 \\
11 & 5.7232 & 0.521 & 5.745 & 0.508 & -0.4 \\
\hline
\end{tabular}

\begin{tabular}{|l|l|l|l|l|l|}
\hline 12 & 5.2318 & 0.573 & 5.250 & 0.520 & -0.4 \\
13 & 6.1669 & 0.469 & 6.190 & 0.478 & -0.4 \\
14 & 6.3485 & 0.495 & 6.365 & 0.480 & -0.3 \\
15 & 6.5604 & 0.495 & 6.570 & 0.480 & -0.2 \\
\hline
\end{tabular}

As can be seen from the table, the two methods measure of the impact force with high consistency, the maximum deviation within $1 \%$. And the rise time difference within $0.05 \mathrm{~ms}$. Put another way to describe. In Figure 7, the two lines can be seen in almost completely overlap

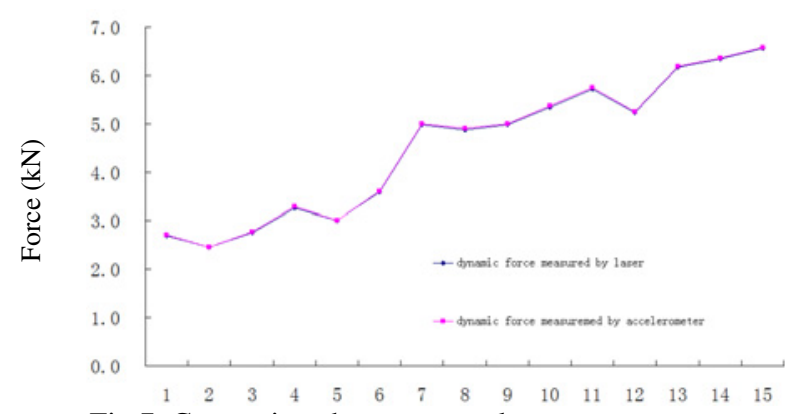

Fig.7. Comparison between accelerator measurement and laser measurement

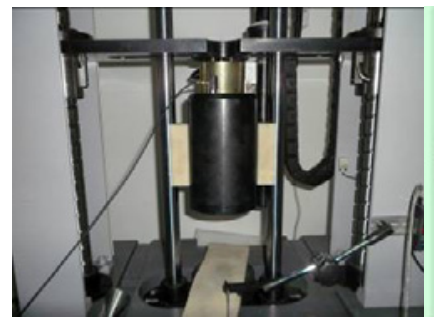

(a)

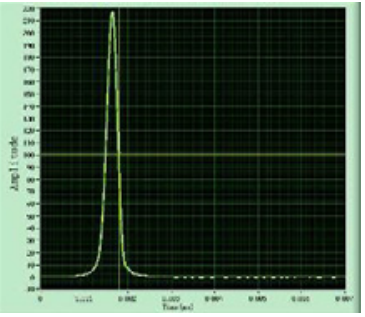

(b)
Fig.8. Accelerometer was mounted on hammer

The (a) of fig 8 is the real picture of experiment, where the standard accelerometer was mounted on the top of large hammer. The (b) of fig 8 show us two curves. White curve is the dynamic force curve of accelerometer measurement. Green curve is the dynamic force curve of laser measurement. From the figure we can see, Two curves basically coincide.

\subsection{Calibration of force transducer by impact excitation}

We carried out several experiments with different types of force sensor. HBM Company C2-20kN force sensor as an example, its technical indicators Accuracy class 0.1, linear error is less than $0.1 \%$, since the vibration frequency is $13 \mathrm{kHz}$. We have done the natural frequency test of this sensor, the results shown in Figure 9:

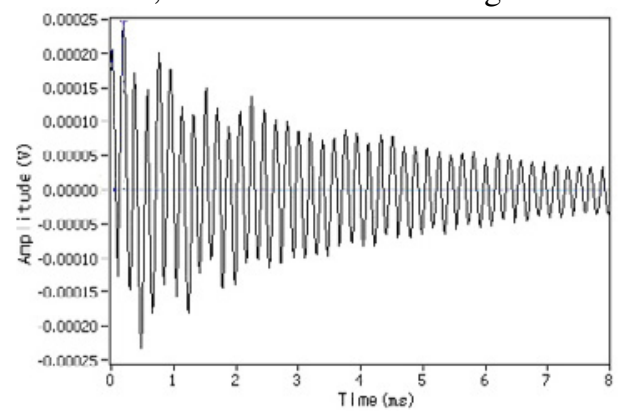

Fig .9 the domain vibration waveform of strain force sensor 
Its natural frequency corresponds with factory specifications that can do the dynamic force test which pulse width larger than $0.1 \mathrm{~ms}$. First, the sensor force standard machine for static calibration, given its static characteristics in line with the factory index, linearity error, repeatability error are better than $0.1 \%$

The sensor is mounted on the laser calibration standard equipment for impact dynamic forces calibration. Results are shown in Table 2:

Table 2

\begin{tabular}{|c|c|c|}
\hline $\begin{array}{c}\text { Standard } \\
\text { force(N) }\end{array}$ & $\begin{array}{c}\text { Indication of force } \\
\text { transducer }(\mathrm{N})\end{array}$ & $\begin{array}{c}\text { Indication error } \\
(\%)\end{array}$ \\
\hline 8868 & 8917 & 0.6 \\
8736 & 8842 & 1.3 \\
10428 & 10610 & 1.8 \\
10736 & 10836 & 1.0 \\
12262 & 12474 & 1.8 \\
12452 & 12575 & 1.0 \\
13821 & 13967 & 1.1 \\
13883 & 14036 & 1.2 \\
14551 & 14815 & 1.9 \\
15023 & 15287 & 1.8 \\
\hline
\end{tabular}

Fig 10 is the real picture of force transducer calibrated by the impact force standard equipment.

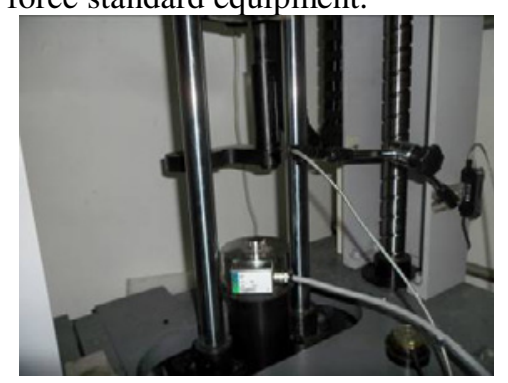

Fig.10. force transducer calibration

\section{Conclusions}

The dynamic force measurements directly traceable to the mass, length and time of three basic quantities, by laser dynamic force measurement, thus avoiding the uncertainty introduced by intermediate links, the dynamic force measurement uncertainty further improved. Still Need for some researches in future. One is the acceleration distribution of the hammer in impact process, and studies its effect on the dynamic force calibration. The other research is effect of hammer natural frequency. Because high-speed impact, the broadband dynamic force can stimulate the natural frequency of drop hammer, so that the distortion of dynamic forces waveform occurred.

\section{References}

1. Zhang Yue, $1 \mathrm{MN}$ dynamic force calibration equipment. Proceedings of the $5^{\text {th }}$ Asia-Pacific Symposium on measurement of force, mass and torque(APMF2000) , 9 (2000)

2. Tomas Bruns. Michael kobusch, Impulse Force Calibration: Design and simulation of a new calibration device, Proceedings of the 17th international Conference on Force, Mass, Torque and Pressure Measurements, IMEKO TC3,17-21 Sept.2001,Istanbut,Turkey

3. Michael kobusch. Tomas Bruns, THE NEW IMPACT FORCE MACHINE AT PTB, XVII IMEKO World Congress Metrology in the 3rd Millennium June 22-27, 2003, Dubrovnik, Croatia.

4. Chinese Verification Regulation: Dynamic Force Transducer

5. Yusaku Fujii, Toward dynamic force calibration, measurement (42) 2009 1039-1044

6. Michael Kobusch, Thomas Bruns,UNCERTAINTY CONTRIBUTIONS OF THE IMPACT FORCE MACHINE AT PTB, XVIII IMEKO WORLD CONGRESS Metrology for a Sustainable Development September, 17 - 22, 2006, Rio de Janeiro, Brazil

7. Meng feng, A NEW METHOD FOR DYNAMIC FORCE MEASUREMENT AT NIM, IMEKO 2010 TC3, TC5 and TC22 Conferences Metrology in Modern Context November 22-25, 2010, Pattaya, Chonburi, Thailand 